\title{
INTERFACE DYNAMICS FOR AN ANISOTROPIC ALLEN-CAHN EQUATION
}

\author{
MICHAL BENEŠ \\ Department of Mathematics, Czech Technical University of Prague \\ Trojanova 13, 12000 Prague, Czech Republic \\ E-mail:Benes@km1.fjfi.cvut.cz \\ DANIELLE HILHORST \\ Laboratoire de Mathématique, Université de Paris Sud \\ 91405 Orsay Cedex, France \\ E-mail: Danielle.Hilhorst@math.u-psud.fr \\ RÉMI WEIDENFELD \\ Ecole Centrale de Lyon, Département Math-Info \\ 36, Avenue Guy de Collongue, 69134 Ecully Cedex, France \\ E-mail: Remi.Weidenfeld@ec-lyon.fr
}

1. Introduction. Main results. The purpose of this work is to study the limiting behavior as $\epsilon \rightarrow 0$ of the solution $u^{\epsilon}$ of Problem $\left(P^{\epsilon}\right)$,

$$
\left(P^{\epsilon}\right) \begin{cases}u_{t}=\operatorname{div}\left(\Phi^{0}(\nabla u) \Phi_{\xi}^{0}(\nabla u)\right)-F \tilde{\Phi}^{0}(\nabla u)+\frac{1}{\epsilon^{2}} f(u) & \text { in } \Omega \times(0, \infty), \\ \nabla_{\Phi} u . \nu=0 & \text { on } \partial \Omega \times(0, \infty) \\ u(x, 0)=u_{0}(x) & \text { for all } x \in \Omega\end{cases}
$$

where $\Omega$ is a smooth bounded domain of $\mathbb{R}^{N}(N \geq 2), \nu$ is the Euclidean unit normal vector exterior to $\partial \Omega, f(s)=2 s\left(1-s^{2}\right), F=F(x, t)$ is a smooth function on $\bar{\Omega} \times(0, \infty)$, $\Phi$ and $\tilde{\Phi}$ are two Finsler metrics with dual functions $\Phi^{0}$ and $\tilde{\Phi}^{0}$ respectively and where $\Phi_{\xi}^{0}$ denotes the gradient of $\Phi^{0}$. For a precise definition of Finsler metric and Finsler geometry, we refer to [2] or to [3].

2000 Mathematics Subject Classification: Primary 35B25, 82C26; Secondary 35K55, 80A22, $65 \mathrm{~N} 40,53 \mathrm{C} 80$.

The paper is in final form and no version of it will be published elsewhere. 
Problem $\left(P^{\epsilon}\right)$ has been proposed by M. Beneš; the partial differential equation in $\left(P^{\epsilon}\right)$ represents an anisotropic version of the equation

$$
u_{t}=\Delta u-F|\nabla u|+\frac{1}{\epsilon^{2}} f(u),
$$

which has been analyzed by M. Beneš and K. Mikula in the context of mean curvature flow, and by M. Beneš in the context of microstructure formation during solidification (we refer to [3] for the references). In particular, they have remarked that the gradient term in (1) allows to construct a better numerical scheme than the usual form of the Allen-Cahn equation

$$
u_{t}=\Delta u+\frac{1}{\epsilon^{2}}\left(f(u)+\frac{\epsilon}{c^{\prime}(0)} F\right),
$$

where $c$ is the velocity of a related travelling wave.

In our work, we make the following hypotheses on $\Phi^{0}$ and $\tilde{\Phi}^{0}$ :

$$
H_{\Phi} \quad\left\{\begin{array}{l}
\Phi^{0} \in C^{3+\alpha}\left(\mathbb{R}^{N} \backslash\{0\}\right), \\
\tilde{\Phi}^{0} \in C^{1+\alpha}\left(\mathbb{R}^{N} \backslash\{0\}\right),
\end{array}\right.
$$

for some $\alpha \in(0,1)$. Setting $\Gamma_{0}:=\left\{x \in \Omega, u_{0}(x)=0\right\}$, we assume that

$$
H_{0}\left\{\begin{array}{l}
u_{0} \in C^{2}(\bar{\Omega}) \text { satisfies the compatibility condition } \nabla_{\Phi} u_{0} \cdot \nu=0 \text { on } \partial \Omega, \\
\Gamma_{0} \text { is a } C^{4+\alpha} \text { hypersurface without boundary such that } \Gamma_{0} \subset \subset \Omega, \\
\nabla u_{0} \neq 0 \text { on } \Gamma_{0}, u_{0} \text { is negative inside } \Gamma_{0} \text { and positive outside. }
\end{array}\right.
$$

Moreover, since $\Phi^{0}$ and $\tilde{\Phi}^{0}$ are Finsler metrics, they satisfy the following regularity properties,

$$
\left\{\begin{array}{l}
\Phi^{0} \in C^{0,1}\left(\mathbb{R}^{N}\right),\left(\Phi^{0}\right)^{2} \in C^{1}\left(\mathbb{R}^{N}\right) \\
\left(\Phi^{0}\right)^{2} \text { is strictly convex } \\
\tilde{\Phi}^{0} \in C^{0,1}\left(\mathbb{R}^{N}\right)
\end{array}\right.
$$

We associate to Problem $\left(P^{\epsilon}\right)$ the moving boundary problem

$$
\left(P^{0}\right)\left\{\begin{array}{l}
V_{n, \Phi}=-(N-1) \kappa_{\Phi}+F \frac{\tilde{\Phi}^{0}(\vec{n})}{\Phi^{0}(\vec{n})} \\
\left.\Gamma_{t}\right|_{t=0}=\Gamma_{0},
\end{array} \text { on } \Gamma_{t}, t \in(0, T],\right.
$$

where $V_{n, \Phi}$ is the velocity in the anisotropic normal direction, $\kappa_{\Phi}$ is the anisotropic mean curvature and $\vec{n}$ is the Euclidean normal vector to $\Gamma_{t}$.

For each $t \in[0, T]$, we define $\Omega_{t}^{-}$as the interior of $\Gamma_{t}, \Omega_{t}^{+}$as the exterior of $\Gamma_{t}$ and we set

$$
u(., t)=\left\{\begin{array}{ll}
+1 & \text { in } \Omega_{t}^{+} \\
-1 & \text { in } \Omega_{t}^{-}
\end{array} \quad \text { for } t \in[0, T]\right.
$$

To begin with we prove the existence and uniqueness of a smooth solution of Problem $\left(P^{0}\right)$ locally in time.

Theorem 1. Let hypotheses $H_{\Phi}$ and $H_{0}$ be fulfilled. Then there exists $T>0$ such that Problem $\left(P^{0}\right)$ has a unique solution $\Gamma=\cup\left\{\Gamma_{t} \times\{t\}, 0 \leq t \leq T\right\} \in C^{3+\alpha,(3+\alpha) / 2}$. 
Our second main result deals with the asymptotic behavior of the solution $u^{\epsilon}$ of Problem $\left(P^{\epsilon}\right)$ as $\epsilon$ tends to zero.

ThEOREM 2. Suppose that the hypotheses $H_{\Phi}$ and $H_{0}$ are satisfied and let $T$ be the maximum time of existence of a smooth solution of Problem $\left(P^{0}\right)$. Then as $\epsilon \rightarrow 0, u^{\epsilon}$ converges to $u$ almost everywhere in $\cup\left\{\Omega_{t}^{ \pm} \times\{t\}, t \in(0, T]\right\}$.

Convergence results to viscosity solutions have been obtained by [6] and [7] but they hold for a very limited class of initial conditions.

Sketches of the proofs of Theorems 1 and 2 are presented in Section 2; we refer the reader to [3] for the detailed proofs. In Section 3 we discuss the special case of space dimension 2 .

2. Sketches of the proofs. The proofs of the theorems 1 and 2 follow and extend similar proofs presented in [4] and [5] in the isotropic case.

2.1. Sketch of the proof of Theorem 1. The idea is to rewrite Problem $\left(P^{0}\right)$ as a parabolic partial differential equation on a fixed $\mathbb{R}^{N-1}$ manifold.

To that purpose, we choose a closed $N-1$ manifold $\mathcal{M}$ without boundary that is diffeomorphic to $\Gamma_{0}$. Let $X^{0}: \mathcal{M} \rightarrow \Gamma_{0}$ be a smooth diffeomorphism and denote by $s^{\prime}=\left(s_{1}, \ldots, s_{N-1}\right)$ the coordinates in $\mathcal{M}$. Then each point $x$ in a tubular neighborhood of $\Gamma_{0}$ may be uniquely written in the form $x=X^{0}\left(s^{\prime}\right)+s_{N} \vec{n}$ where $\vec{n}$ is the Euclidean unit normal vector to $\Gamma_{0}$ at the point $X^{0}\left(s^{\prime}\right)$ and $s_{N} \in \mathbb{R}$. In a neighborhood of $\Gamma_{0}$, we perform a coordinate transformation from the usual coordinates $\left(x_{1}, \ldots, x_{N}\right)$ to the local coordinates $\left(s_{1}, \ldots, s_{N}\right)$.

Therefore, solving Problem $\left(P^{0}\right)$ for small time amounts to finding a function $\Lambda$ : $\mathcal{M} \times[0, T] \rightarrow \mathbb{R}$ such that

$$
\Gamma_{t}=\left\{x=X^{0}\left(s^{\prime}\right)+s_{N} \vec{n}, \quad s_{N}=\Lambda\left(s^{\prime}, t\right)\right\} .
$$

The function $\psi=s_{N}-\Lambda$ is such that $\psi<0$ on $\Omega_{t}^{-}, \psi=0$ on $\Gamma_{t}$ and $\psi>0$ on $\Omega_{t}^{+}$. It turns out that $\Lambda$ satisfies the partial differential equation,

$$
\Lambda_{t}=\sum_{i, j=1}^{N-1} a_{i j} \frac{\partial^{2} \Lambda}{\partial s_{i} \partial s_{j}}+\Pi\left(s^{\prime}, t, \Lambda, \nabla \Lambda\right) \quad(l o c),
$$

where $\Pi$ is a smooth function and $a_{i j}$ is defined by

$$
a_{i j}=\vec{\alpha}_{i}\left(s^{\prime}\right) \cdot\left(\Phi^{0} \Phi_{\xi \xi}^{0}\right)(\vec{n}+\vec{G}(\nabla \Lambda)) \vec{\alpha}_{j}\left(s^{\prime}\right) ;
$$

here $\left\{\vec{\alpha}_{i}\right\}_{1 \leq i \leq N-1}$ are smooth vector fields given by the partial derivatives of the diffeomorphism $X^{0}$ and such that $\vec{\alpha}_{i} \neq 0, \vec{\alpha}_{i} \perp \vec{n}$, and $\vec{G}$ is a smooth vector field such that $\vec{G} \perp \vec{n}$.

The last step consists in proving that the equation (loc) is parabolic. To that purpose, we use convexity properties of $\Phi^{0}$ to describe the eigenvalues and the corresponding eigenfunctions of the matrix $\Phi^{0} \Phi_{\xi \xi}^{0}$. We prove that there exists $\bar{\lambda}>0$ such that for all $\xi, \zeta \in \mathbb{R}^{N} \backslash\{0\}$

$$
\zeta \cdot\left(\Phi^{0} \Phi_{\xi \xi}^{0}\right)(\xi) \zeta \geq \bar{\lambda}\left(|\zeta|^{2}-\frac{(\zeta \cdot \xi)^{2}}{|\xi|^{2}}\right) .
$$


Note that if $\Phi^{0}$ is the Euclidean metric then $\left(\Phi^{0} \Phi_{\xi \xi}^{0}\right)(\xi) \zeta=\zeta-\frac{\zeta . \xi}{|\xi|^{2}} \xi$ which is the projection of $\zeta$ on $(\xi)^{\perp}$. It follows from the orthogonality of $\vec{\alpha}_{i}$ and $\vec{G}$ with $\vec{n}$ that for all $\zeta \in$ $\mathbb{R}^{N-1} \backslash\{0\}$,

$$
\begin{aligned}
\sum_{i, j=1}^{N-1} a_{i j} \zeta_{i} \zeta_{j} & =\left(\sum_{i=1}^{N-1} \zeta_{i} \vec{\alpha}_{i}\right) \cdot\left(\Phi^{0} \Phi_{\xi \xi}^{0}\right)(\vec{n}+\vec{G}(\nabla \Lambda))\left(\sum_{i=1}^{N-1} \zeta_{i} \vec{\alpha}_{i}\right) \\
& \geq \bar{\lambda}\left(\left|\sum_{i}^{N-1} \zeta_{i} \vec{\alpha}_{i}\right|^{2}-\frac{\left(\sum_{i}^{N-1} \zeta_{i} \vec{\alpha}_{i} \cdot \vec{G}(\nabla \Lambda)\right)^{2}}{1+|\vec{G}(\nabla \Lambda)|^{2}}\right) \\
& \geq \bar{\lambda} \frac{\left|\sum_{i}^{N-1} \zeta_{i} \vec{\alpha}_{i}\right|^{2}}{1+|\vec{G}(\nabla \Lambda)|^{2}} \geq \bar{\lambda}_{0} \frac{|\zeta|^{2}}{1+|\vec{G}(\nabla \Lambda)|^{2}}
\end{aligned}
$$

where the last inequality can be deduced from the fact that $X^{0}$ is a diffeomorphism. Thus the equation (loc) is parabolic as long as $\nabla \Lambda$ remains bounded. Since $\left.\Lambda\right|_{t=0}=0$ it follows that $\left.\nabla \Lambda\right|_{t=0}=0$ and we may apply an existence and uniqueness theorem for solutions of parabolic equations such as in [8] to deduce that there exists $T>0$ and a unique solution of Problem $\left(P^{0}\right)$ on $[0, T]$. The regularity of the solution $\Gamma \in C^{3+\alpha,(3+\alpha) / 2}$ is obtained by a careful analysis of the regularity of the functions which appear in the proof.

2.2. Sketch of the proof of Theorem 2. The proof of Theorem 2 is divided into two steps. To begin with we state a generation of interface result: the function $u^{\epsilon}$ becomes close to \pm 1 outside of a neighborhood of the level set zero of $u_{0}$ at a time of order $\mathcal{O}\left(\epsilon^{2}|\ln \epsilon|\right)$. Then we present a result about the propagation of interface, namely we prove that the solution $u^{\epsilon}$ remains close to the function $u$ on the time interval $\left[\tau^{\epsilon}, T\right]$.

These results are proved by constructing suitable upper and lower solutions. A difficulty comes from the lack of regularity of the second order term, $\operatorname{div}\left(\Phi^{0}(\nabla u) \Phi_{\xi}^{0}(\nabla u)\right)$. More precisely, if $u \in C^{2}(\Omega)$, the hypotheses $H_{\Phi}$ insure that this second order term is well defined and is continuous at each point $x \in \Omega$ where $\nabla u(x) \neq 0$. On the other hand, at a point $x \in \Omega$ where $\nabla u(x)=0$, the application $x \mapsto \Phi^{0}(\nabla u) \Phi_{\xi}^{0}(\nabla u)$ is of class $C^{1}$ for a very limited class of Finsler metrics $\Phi^{0}$ (such as the Euclidean metric). Due to this fact, we have to work with weak solutions and then with weak upper and lower solutions. But it is quite hard to check if a function is a lower or upper solution with the weak definition. We use homogeneity properties of $\Phi^{0}$ and $\Phi_{\xi}^{0}$,

$$
\begin{aligned}
& \Phi^{0}(s \xi)=|s| \Phi^{0}(\xi) \text { for all } s \in \mathbb{R} \text { and } \xi \in \mathbb{R}^{N}, \text { and } \\
& \Phi_{\xi}^{0}(s \xi)=\frac{s}{|s|} \Phi_{\xi}^{0}(\xi) \text { for all } s \neq 0 \text { and } \xi \neq 0,
\end{aligned}
$$

to deduce that the application $x \mapsto \Phi^{0}(\nabla u) \Phi_{\xi}^{0}(\nabla u)$ admits partial derivatives in any direction even for $x \in \Omega$ such that $\nabla u(x)=0$. More precisely,

Lemma. Let $u \in C^{2}(\bar{\Omega})$; then the application $x \mapsto \operatorname{div}\left(\Phi^{0}(\nabla u) \Phi_{\xi}^{0}(\nabla u)\right)$ is well defined and belongs to $L^{\infty}(\Omega)$.

Then proving that a smooth function $u \in C^{2,1}(\bar{\Omega} \times[0,+\infty))$ is an upper or lower solution amounts to check the sign of the expression,

$$
\mathcal{L}^{\epsilon}(u):=u_{t}-\operatorname{div}\left(\Phi^{0}(\nabla u) \Phi_{\xi}^{0}(\nabla u)\right)+F \tilde{\Phi}^{0}(\nabla u)-\frac{2}{\epsilon^{2}} u\left(1-u^{2}\right)
$$


Note that the solution $u^{\epsilon}$ of Problem $\left(P^{\epsilon}\right)$ is not necessarily smooth so that $\mathcal{L}^{\epsilon}\left(u^{\epsilon}\right)$ is not defined. The above criterion is valid only for smooth enough upper and lower solutions. To perform the computations, we extensively use the equality $\Phi_{\xi}^{0}(\xi) \cdot \xi=\Phi^{0}(\xi)$ for $\xi \neq 0$ which is obtained by differentiating the first equality in equation (3).

We construct upper and lower solutions in the following way.

For the generation of interface property: we slightly change the nonlinear function $f(u)=2 u\left(1-u^{2}\right)$ around its unstable zero and denote the resulting function by $f_{\epsilon}$. A basic ingredient is the initial value problem,

$$
\left\{\begin{array}{l}
\frac{\partial w}{\partial \tau}(\zeta, \tau)=f_{\epsilon}(w) \quad \text { for } \tau \in(0, \infty) \\
w(\zeta, 0)=\zeta
\end{array}\right.
$$

where, more precisely, the function $f_{\epsilon}$ satisfies

(i) $f_{\epsilon}(s)=f(s)$ for all $s \in\left(-\infty,-\frac{\epsilon}{4}\right] \cup[3 \epsilon|\ln \epsilon|,+\infty)$,

(ii) $f_{\epsilon}(s) \leq f(s)$ for all $s \in \mathbb{R}$,

(iii) $f_{\epsilon}(s)=\frac{s-\epsilon|\ln \epsilon|}{|\ln \epsilon|}$ for all $s \in[0,2 \epsilon|\ln \epsilon|]$,

(iv) $f_{\epsilon}$ has exactly three zeroes, namely $-1, \epsilon|\ln \epsilon|$ and 1 .

Then for all $\tau_{0}>0$ there exists a constant $C=C\left(\tau_{0}\right)>0$ such that the function $\underline{u}^{\epsilon}(x, t)=$ $w\left(u_{0}-C t / \epsilon, t / \epsilon^{2}\right)$ is a lower solution of Problem $\left(P^{\epsilon}\right)$ on $\left[0, \tau_{\epsilon}\right]$ where $\tau_{\epsilon}=\tau_{0} \epsilon^{2}|\ln \epsilon|$. Moreover, for all $k>0$, there exist $\tau_{0}, c>0$ such that

$$
\begin{cases}\underline{u}^{\epsilon}\left(x, \tau_{\epsilon}\right) \geq-1-\epsilon^{k} & \text { for all } x \in \Omega, \\ \underline{u}^{\epsilon}\left(x, \tau_{\epsilon}\right) \geq 1-\epsilon^{k} & \text { for all } x \in \Omega \text { such that } u_{0}(x) \geq c \epsilon|\ln \epsilon| .\end{cases}
$$

Similarly, we construct an upper solution which satisfies corresponding inequalities. Then at the time $\tau_{\epsilon}$ the solution $u^{\epsilon}$ of Problem $\left(P^{\epsilon}\right)$ is close to \pm 1 outside a tubular neighborhood of the level set $\left\{u_{0}=0\right\}$ of thickness $\mathcal{O}(\epsilon|\ln \epsilon|)$.

For the propagation of interface property: we construct upper and lower solutions on the time interval $\left[\tau_{\epsilon}, T+\tau_{\epsilon}\right]$ where $T$ is the maximum time of existence of a solution of Problem $\left(P^{0}\right)$. The essential ingredients are :

The modified signed anisotropic distance function $d_{\Phi}$ to the interface $\Gamma_{t}$ which is smooth, positive in $\Omega_{t}^{+}$, negative in $\Omega_{t}^{-}$and such that $\left|d_{\Phi}(x, t)\right|$ is the anisotropic distance to $\Gamma_{t}$ in a tubular neighborhood with thickness $d_{0}>0$ of $\Gamma$ and is a constant outside a tubular neighborhood with thickness $2 d_{0}$ of $\Gamma$. Two important properties of $d_{\Phi}$ are

$$
\begin{cases}\Phi^{0}\left(\nabla d_{\Phi}\right)=1 & \text { in a neighborhood of } \Gamma, \\ \left(d_{\Phi}\right)_{t}=\Delta_{\Phi} d_{\Phi}-F \tilde{\Phi}^{0}\left(\nabla d_{\Phi}\right) & \text { on } \Gamma .\end{cases}
$$

The travelling wave solutions $U(z, \delta)$ of a family of related one dimensional parabolic equations indexed by $\delta(|\delta|$ is small). More precisely, $U(., \delta)$ is the solution of

$$
\left\{\begin{array}{l}
U_{z z}+c(\delta) U_{z}+2 U\left(1-U^{2}\right)+\delta=0 \quad z \in \mathbb{R}, \\
U(-\infty)=h_{-}(\delta), U(\infty)=h_{+}(\delta),
\end{array}\right.
$$

where $h_{-}(\delta), h_{0}(\delta)$ and $h_{+}(\delta)$ are the three roots of the polynomial $2 u\left(1-u^{2}\right)+\delta=0$ in increasing order. Since Problem $(Q)$ is of bistable type, there exists a unique $c=c(\delta)$ 
and a unique solution $U=U(z, \delta)$ up to translations. Note that $c(0)=0$ and that the function $\delta \mapsto c(\delta)$ is smooth. Moreover, $U$ is increasing in $\mathbb{R}$ and $U-h_{ \pm}$, as well as its derivatives, decay exponentially fast at infinity.

Using these properties, we show that the functions $\bar{u}^{\epsilon}$ and $\underline{u}^{\epsilon}$ defined by

$$
\begin{aligned}
& \bar{u}^{\epsilon}\left(x, t+\tau_{\epsilon}\right)=U\left(\frac{d_{\Phi}(x, t)+m_{1} \epsilon|\ln \epsilon| e^{m_{2} t}}{\epsilon}, \epsilon^{2} m_{3} e^{m_{2} t}\right), \\
& \underline{u}^{\epsilon}\left(x, t+\tau_{\epsilon}\right)=U\left(\frac{d_{\Phi}(x, t)-m_{1} \epsilon|\ln \epsilon| e^{m_{2} t}}{\epsilon},-\epsilon^{2} m_{3} e^{m_{2} t}\right),
\end{aligned}
$$

for constants $m_{1}, m_{2}, m_{3}>0$, are upper and lower solutions for Problem $\left(P^{\epsilon}\right)$ on $\left[\tau_{\epsilon}, \tau_{\epsilon}+T\right]$. Note that $\bar{u}^{\epsilon}$ and $\underline{u}^{\epsilon}$ tend to $u$ as $\epsilon \rightarrow 0$.

3. The case of space dimension 2. An essential application is a generic anisotropy in space dimension 2. It has the form

$$
\Phi^{0}(\xi)=|\xi| \Psi_{0}\left(\frac{\xi}{|\xi|}\right)
$$

where $\Psi_{0}$ is a smooth function. If $\Psi(\theta):=\Psi_{0}\left(\left(\begin{array}{c}\cos \theta \\ \sin \theta\end{array}\right)\right)=\Phi^{0}\left(\left(\begin{array}{c}\cos \theta \\ \sin \theta\end{array}\right)\right)$, the convexity of $\Phi^{0}$ implies that $\Psi+\Psi^{\prime \prime}>0$ and the corresponding motion law $\left(P^{0}\right)$ has the form

$$
\left(\mathcal{P}^{0}\right) \quad\left\{\begin{array}{l}
V_{n}=-(N-1) \Psi(\theta)\left(\Psi(\theta)+\Psi^{\prime \prime}(\theta)\right) \kappa+F \tilde{\Psi}(\theta) \quad \text { on } \Gamma_{t}, t \in(0, T] \\
\left.\Gamma_{t}\right|_{t=0}=\Gamma_{0},
\end{array}\right.
$$

where $V_{n}$ is the velocity in the Euclidean normal direction, $\kappa$ is the usual mean curvature of $\Gamma_{t}, \theta=\theta(x, t)$ is the angle of the Euclidean normal vector to $\Gamma_{t}$ at the point $x$ with the $x_{1}$-axis and $\tilde{\Psi}(\theta):=\tilde{\Phi}^{0}\left(\left(\begin{array}{c}\cos \theta \\ \sin \theta\end{array}\right)\right)$.

Problem $\left(\mathcal{P}^{0}\right)$, which arises in the description of phase transitions between liquid and solid phases in the context of Stefan problems, has been extensively studied by [1]. A consequence of our results is that Problem $\left(\mathcal{P}^{0}\right)$ coincides with the singular limit as $\epsilon$ tends to zero of Problem $\left(\mathcal{P}^{\epsilon}\right)$

$$
\left(\mathcal{P}^{\epsilon}\right) \begin{cases}u_{t}=\Delta_{\Phi} u-F|\nabla u| \tilde{\Psi}_{0}\left(\frac{\nabla u}{|\nabla u|}\right)+\frac{1}{\epsilon^{2}} f(u) & \text { in } \Omega \times(0, \infty), \\ \nabla_{\Phi} u . \nu=0 & \text { on } \partial \Omega \times(0, \infty), \\ u(x, 0)=u_{0}(x) & \text { for all } x \in \Omega,\end{cases}
$$

where $\tilde{\Psi}_{0}\left(\left(\begin{array}{c}\cos \theta \\ \sin \theta\end{array}\right)\right):=\tilde{\Psi}(\theta)$ and where the operator $\Delta_{\Phi}$ is given by

$$
\Delta_{\Phi} u:=\operatorname{div} \nabla_{\Phi} u
$$

with

$$
\nabla_{\Phi} u:=\Psi_{0}^{2}\left(\frac{\nabla u}{|\nabla u|}\right) \nabla u-\Psi_{0}\left(\frac{\nabla u}{|\nabla u|}\right) \Psi_{0}^{\prime}\left(\frac{\nabla u}{|\nabla u|}\right)(\nabla u)^{\perp},
$$

and $\xi^{\perp}=\left(\xi_{2},-\xi_{1}\right)$. 


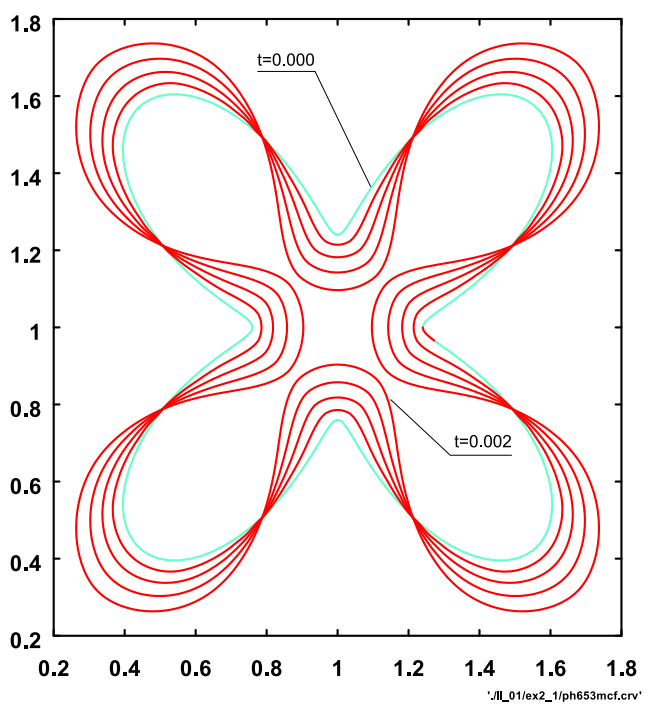

Fig. 1. Motion of an interface satisfying $V_{n}=-\Psi(\theta)\left(\Psi(\theta)+\Psi^{\prime \prime}(\theta)\right) \kappa+\left.F\right|_{\Gamma}$, obtained by solving the reaction-diffusion equation in Problem $\left(\mathcal{P}^{\epsilon}\right)$.

\section{References}

[1] S. Angenent and M. E. Gurtin, Multiphase thermomechanics with interfacial structure. II. Evolution of an isothermal interface, Arch. Rational Mech. Anal. 108 (1989), 323-391.

[2] G. Bellettini and M. Paolini, Anisotropic motion by mean curvature in the context of Finsler geometry, Hokkaido Math. J. 25 (1996), 537-566.

[3] M. Beneš, D. Hilhorst and R. Weidenfeld, Interface dynamics for an anisotropic Allen-Cahn equation, in preparation.

[4] X. Chen, Generation and propagation of interfaces for reaction-diffusion equations, J. Differential Equations 96 (1992), 116-141.

[5] X. Chen and F. Reitich, Local existence and uniqueness of solutions of the Stefan problem with surface tension and kinetic undercooling, J. Math. Anal. Appl. 164 (1992), 350-362.

[6] C. M. Elliott, M. Paolini, and R. Schätzle, Interface estimates for the fully anisotropic Allen-Cahn equation and anisotropic mean-curvature flow, Math. Models Methods Appl. Sci. 6 (1996), 1103-1118.

[7] C. M. Elliott and R. Schätzle, The limit of the fully anisotropic double-obstacle Allen-Cahn equation in the nonsmooth case, SIAM J. Math. Anal. 28 (1997), 274-303.

[8] O. A. Ladyženskaja, V. A. Solonnikov, and N. N. Ural'ceva, Linear and quasilinear equations of parabolic type, Translated from the Russian by S. Smith, Vol. 23, Translations of Mathematical Monographs, American Mathematical Society, Providence, R.I., 1967. 